\title{
Selladores de Superficie en Base a Resina: Potencial de Prevenir Tinción Exógena
}

\author{
Resin-based Surface Sealants: Potential to Prevent Exogenous Staining
}

Pablo Angel Aguirre'; Andrea Gallegos Fauré2; Cristian Bersezio Miranda'; Juan Estay Larenas ${ }^{1}$ \& Roque Arias Fredes ${ }^{1,2}$

\begin{abstract}
AGUIRRE, P. A.; GALLEGOS, F. A.; BERSEZIO, M. C.; ESTAY, L. J. \& ARIAS, F. R. Selladores de superficie en base a resina: potencial de prevenir tinción exógena. Int. J. Odontstomat., 12(4):348-354, 2018.

RESUMEN: El objetivo del presente trabajo fue estudiar el potencial de los selladores de superficie basados en resinas de evitar cambios de color de resinas compuestas de restauración ante la acción de una bebida coloreada. Para ello, se confeccionó una muestra de 40 discos de resina nanocompuesta Filtek Z350 XT (3M- ESPE) color A2D de 7x2 mm, se pulieron en forma convencional con una serie de discos de óxido de aluminio y se distribuyeron aleatoriamente en 2 grupos de 20 especímenes. Al grupo 1 se le selló toda su superficie usando el agente sellador Fortify ${ }^{\circledR}$, al grupo 2 se le dejó sin tratar. Se sometió a todos los discos $(n=40)$ a un proceso de termociclado luego del cual se procedió a medir su color inicial mediante el espectrofotómetro de punto Vita Easyshade $\mu^{\circledR}$. Cada grupo de discos fue inmerso en recipientes metálicos de acero inoxidable en un medio que contenía a una infusión concentrada de té negro durante 7 días a $37{ }^{\circ} \mathrm{C}$. Finalmente, se midió el color final a cada uno de los grupos de discos luego del periodo de inmersión. Los resultados fueron sometidos al análisis estadístico con el programa Stata 12 y el test T-Student con una significancia de $p<0,05$. Los resultados indicaron un significativo cambio de color de ambos grupos de discos, siendo ligeramente menor en el grupo de discos sellados, sin embargo, no existió diferencia significativa entre ellos. Como conclusión se determinó que bajo las condiciones del presente estudio el uso del agente sellante de superficie Fortify ${ }^{\circledR}$ no evitó el cambio de color de la resina nanocompuesta de restauración.
\end{abstract}

PALABRAS CLAVE: resinas compuestas, sellantes, color.

\section{INTRODUCCIÓN}

El uso de resinas compuestas como material restaurador se ha incrementado crecientemente debido a sus excelentes propiedades clínicas. A pesar de mejoras en las formulaciones, la estabilidad de color de las resinas después de exposición al medio oral por largo plazo constituye una permanente preocupación (Guler et al., 2005).

El cambio de color de las restauraciones estéticas es una razón común de su reemplazo (Leibrock et al., 1997). Tres tipos de alteraciones de color han sido descritas en la literatura: discoloración externa debido a acumulación de placa y tinciones superficiales; alteraciones en la superficie o sub superficie resultando en una degradación superficial o en una ligera penetración y adsorción de agentes colorantes o tincionantes en la capa superficial de las resi- nas compuestas y, descoloraciones intrínsecas debido a reacciones fisicoquímicas en la matriz del composite en las capas superficiales y más profundas del material, gatilladas por radiación UV, energía térmica o humedad (Dietschi et al.,1994; Leibrock et al.).

La estabilidad de color de las resinas compuestas puede ser influenciada por fuentes intrínsecas y extrínsecas. Los factores extrínsecos incluyen intensidad y duración de la polimerización, exposición a factores ambientales, incluyendo radiación ambiental y UV, calor, agua y colorantes alimentarios. Los factores intrínsecos incluyen composición de la matriz de resina, relleno, carga y distribución de las partículas, tipo de foto iniciador y porcentaje remanente de doble enlaces (Sarafianou et al., 2007; Ren et al., 2012).

\footnotetext{
${ }^{1}$ Facultad de Odontología, Universidad de Chile, Santiago, Chile.

${ }^{2}$ Facultad de Medicina CAS, Odontología, Universidad del Desarrollo, Santiago, Chile.
} 
La discoloración puede evaluarse mediante mecanismos visuales e instrumentales. Las técnicas instrumentales eliminan la interpretación subjetiva de la comparación visual del color. La diferencia entre un color y otro se expresa a través de su variación total de color (DE), reportándose recientemente en un estudio clínico que un valor de DE 2,6 es el valor en que el $50 \%$ de los dentistas pueden percibir una diferencia (50/50 perceptibilidad) y un valor de DE 5,5 el valor en que el $50 \%$ de dentistas reharían la restauración (50/50 aceptabilidad) (Douglas et al., 2009). En principio, ninguna diferencia de color $(\mathrm{DE}=0)$ indica un material con estabilidad de color completa o sin coloración (Guler et al.). 10 La tinción de materiales a base de resina por enjuagues bucales, café, té, vino tinto, jugos, agua y luz UV que simula la exposición intraoral a largo plazo ha sido reportada (Bagheri et al., 2005; Guler et al.; Ertas et al., 2006; Omata et al., 2006; Catelan et al., 2011; Subramanya \& Muttagi, 2011; Ren et al.; Kumari et al., 2015).

El acabado y el pulido de las restauraciones dentales son pasos esenciales en el proceso restaurativo que mejoran la estética y la longevidad de las restauraciones de resina compuesta (Sarac et al., 2006). El uso de resinas sin relleno para resinas compuestas se sugirió por primera vez a fines de los años ochenta en un intento de llenar estos defectos microestructurales y mejorar Resistencia a la abrasión y tinción reduciendo la porosidad superficial (Sarac et al.; Singh et al., 2015).

De los pocos estudios existentes, al evaluar la resistencia a la tinción usando selladores de superficie se ha visto que éstos pueden mejorar la resistencia a tinción de materiales de restauración en base a resina compuesta (Doray et al., 2003). Por el contrario, Lee \& Powers (2007) al evaluar el uso de sellante superficial y el efecto combinado de mucina, clorhexidina y solución de té, en la tinción de cuatro resinas compuestas, no observaron diferencias entre las resinas compuestas selladas y las no selladas. Soares et al., observaron la influencia de la unidad de curado y la protección del sellante superficial sobre las resinas compuestas teñidas por el café, y concluyeron que los selladores superficiales no impiden la tinción del material compuesto (Soares et al., 2007). Catelan et al. observaron que el sellante de superficie no modificaba el efecto de tinciones ni el resultado del envejecimiento artificial mediante luz UV.

El objetivo de este estudio in vitro fue determinar si el sellante de superficie Fortify (BISCO, Inc. 1100
W. Irving Park Rd. Schaumburg, IL 60193 U.S.A.) aumenta la resistencia a la tinción de una resina de nanotecnología. La hipótesis nula fue que el sellante de superficie no aumenta la resistencia a tinción de una resina de nanotecnología.

\section{MATERIAL Y MÉTODO}

Basados en el tamaño muestral del estudio de Sahin et al. (2015), se confeccionaron 40 discos de resina compuesta Filtek Z350 XT, A2 Dentina (3M ESPE) de $7 \times 2 \mathrm{~mm}$. Para ello se usaron matrices de poliuretano translúcido de altura ligeramente mayor a $2 \mathrm{~mm}$. Cada matriz estandarizada se posicionó sobre una loseta estéril. La resina compuesta correspondiente, se empacó con una espátula recubierta con teflón (Espátula A6, Hu-Friedy Mfg.Co, Inc, Chicago, USA) hasta el tope de la matriz. Posteriormente, para evitar la formación de la capa inhibida por oxígeno se colocó sobre ésta un portaobjeto que comprimió el composite a su dimensión final y posteriormente, se fotopolimerizó con lámpara de fotocurado (EliparTM LED, 3M ESPE) durante 1 minuto en cada superficie con el fin de asegurar la correcta polimerización del material. Todos los discos fueron realizados por un solo operador.

Posteriormente, se realizó pulido de las superficies de los discos siguiendo la secuencia completa de discos de óxido de aluminio Sof-lex (3M ESPE), de manera de replicar un protocolo ampliamente usado en clínica con buenos resultados de lisura superficial (Avsar et al., 2015). Los cuarenta discos que cumplieron con las medidas establecidas se asignaron aleatoriamente a dos grupos A y B. Ambos grupos fueron lavados profusamente con agua y secados.

El grupo A correspondió a los discos recubiertos con sellador de superficie. Para esto, cada disco fue cubierto en su totalidad con el producto Fortity (BISCO Inc., IL 60193 U.S.A.) (Tabla I). Siguiendo las instrucciones del fabricante, luego del lavado y secado se procedió a aplicar ácido fosfórico al 37 \% (Scotchbond gel grabador 3M ESPE) en su superficie, se lavo profusamente con agua y secó con aire. Se aplicó una delgada capa del agente sellador usando un movimiento de frotación, se adelgazó la capa con aire y se polimerizó por 10 segundos. Lo mismo se repitió por cada cara.

El grupo B constituyó el grupo de pulido, y sirvió como control. 
Posteriormente, los dos grupos de discos fueron sometidos a termociclado con el fin de emular el comportamiento de una resina compuesta en el medio oral. Se sometieron a 300 ciclos de 30 segundos cada uno en agua a $5{ }^{\circ} \mathrm{C}$ y $55^{\circ} \mathrm{C}$ en una termocicladora Thermo Scientific Modelo 251 (Fisher Scientific SL - C/ Luis I, 9 - 28031 Madrid, España).

Una vez retirados del termociclador, los discos fueron lavados con agua destilada y secados con aire y se procedió a la medición basal de color con un espectrofotómetro de punto configurado para obtener los valores CIE L*a*b (International Commission on Illumination, 1986). De esta manera se obtuvieron los valores de las tres coordenadas $\left(L^{*} a^{*} b^{*}\right)$ para cada grupo de discos, los cuales se denominaron L1 a1 y b1. Para la medición del color, cada disco se posicionó sobre un fondo blanco usando para este fin tarjetas de calibración fotográfica Opteka (Opteka BDWH1 9' X 13' White/Black Muslin Professional Background) (Sun et al., 2011). La medición fue realizada usando el espectrofotómetro digital VITA EasyShade Compact (VITA Zahnfabrik H. Rauter GmbH \& Co., Germany). El aparato fue manejado por un operador calibrado (Kappa > 0.8), el cual repitió 4 veces el procedimiento para evitar variaciones debido al posible posicionamiento inadecuado del espectrofotómetro $u$ otras variables.

Luego los discos de ambos grupos se sometieron a un inmersión en una infusión concentrada de té negro Ceylán Supremo (Supremo,Santiago, Chile). Cada grupo de discos estuvo sumergido en un recipiente metálicos de acero inoxidable por 7 días a $37^{\circ} \mathrm{C}$ en una estufa marca Memmert Modelo 600 ( Memmert GmbH + Co. KG, Aeussere Rittersbacher Strasse 38 D-91126 Schwabach), en un ambiente oscuro. Posteriormente se procedió a lavar los discos de resina por 30 segundos con agua destilada y se secaron con aire. Se midió el color nuevamente mediante el espectofotómetro de punto obteniendo los valores L2 a2 y b2 para cada grupo.

Los datos recolectados fueron ingresados a una planilla del programa Excel Office. La variación de color de cada disco, fue obtenida mediante el cálculo de Delta $E(D E)$ de acuerdo a la siguiente fórmula:

$$
\mathrm{DE}=\left[(\mathrm{L} 1-\mathrm{L} 2) \sum+(\mathrm{a} 1-\mathrm{a} 2) \sum+(\mathrm{b} 1-\mathrm{b} 2) \sum\right] 1 / 2
$$

Se calculó un promedio de Delta E de todos los discos del grupo A y B y se compararon entre ellos mediante test $t$ de Student utilizando el programa estadístico Stata 12 (StataCorp LLC, 4905 Lakeway Drive, College Station, Texas 778454512 ,USA). Se fijó un nivel de significancia de un 5 $\%$ (intervalo de confianza de $95 \%$ ) y un valor de $p<$ 0,05 .

Tabla I. Composición de materiales usados.

\begin{tabular}{lll}
\hline Material & Fabricante & $\begin{array}{l}\text { Composición } \\
\text { (manufacturer } \\
\text { SDS) }\end{array}$ \\
\hline $\begin{array}{l}\text { Fortify (sellante de } \\
\text { superficie sin relleno) }\end{array}$ & $\begin{array}{l}\text { Bisco Inc, } \\
\text { USA }\end{array}$ & $\begin{array}{l}\text { UDMA } \\
\text { (dimetacrilato de } \\
\text { uretano) } \\
\text { Bis-GMA, UDMA, } \\
\text { TEGDMA, Bis- } \\
\text { Filtek Z350 XT }\end{array}$ \\
3M ESPE, PEGDMA, \\
USA & \begin{tabular}{l} 
silica, zirconia \\
\hline
\end{tabular} \\
\hline
\end{tabular}

\section{RESULTADOS}

Los resultados obtenidos de las mediciones espectrofotométricas se tabularon para cada uno de los discos de cada grupo.

En la Tabla II se aprecia los resultados obtenidos por los discos del Grupo A (discos sellados en superficie con $\mathrm{Fortify}^{\circledR}$ ). Se puede apreciar variación de los valores CIELab en amplio rango, apreciándose disminución del valor $L$ y aumento valores $a^{*} y b^{*}$. De igual manera los discos del Grupo B (Discos sin sellado superficial) experimentaron similares cambios (Tabla III).

En la Tabla IV se puede apreciar la diferencia de color experimentada por ambos grupos después de ser sometidos a la inmersión en té. Se puede ver que los valores promedio de DE de cada grupo varían entre 7,309 y 7,657 con un valor de P-Value aplicando el test de T-Student de 0,674, evidenciando diferencia de DE no significativa estadísticamente.

\section{DISCUSIÓN}

Los sellantes de superficie de baja viscosidad son usados para mejorar la resistencia a la abrasión y la integridad de las restauraciones penetrando las microporosidades en la superficie de la restauración. 
AGUIRRE, P. A.; GALLEgos, F. A.; BERSEZIO, M. C.; ESTAY, L. J. \& ARIAS, F. R. Selladores de superficie en base a resina: potencial de prevenir tinción exógena. Int. J. Odontstomat., 12(4):348-354, 2018.

Tabla II. Variación de color en coordenadas $L^{*}, a^{*}, b^{*}$ de discos de resina del grupo A.

\begin{tabular}{|c|c|c|c|c|c|c|c|}
\hline \multicolumn{8}{|c|}{ Grupo A } \\
\hline \multirow{2}{*}{ Disco } & \multicolumn{3}{|c|}{ Color Basal } & \multicolumn{3}{|c|}{ Color después de inmersión en té } & \multirow{2}{*}{$\Delta \mathbf{E}$} \\
\hline & L1 & a1 & b1 & L2 & a2 & b2 & \\
\hline 1 & 76,1 & 2,9 & 37,9 & 74,8 & 5,4 & 41,7 & 4,73 \\
\hline 2 & 81 & 2,4 & 38,8 & 77,8 & 4,3 & 41,5 & 4,60 \\
\hline 3 & 81,3 & 2,2 & 39 & 75,3 & 5 & 43,4 & 7,95 \\
\hline 4 & 82 & 3,9 & 39,7 & 74,4 & 4,9 & 43 & 8,35 \\
\hline 5 & 83,3 & 2,5 & 39,6 & 73,9 & 6,2 & 46,1 & 12,01 \\
\hline 6 & 79,3 & 3,5 & 41,4 & 75,7 & 4,7 & 43,3 & 4,24 \\
\hline 7 & 81,7 & 3,3 & 39,1 & 69,2 & 7,5 & 40,5 & 13,26 \\
\hline 8 & 82,5 & 2,7 & 39,5 & 71,9 & 6,6 & 41,7 & 11,51 \\
\hline 9 & 80,9 & 4,1 & 39,7 & 75,3 & 4,7 & 39,9 & 5,64 \\
\hline 10 & 80,9 & 2,4 & 38,4 & 73,8 & 5,6 & 42,1 & 8,62 \\
\hline 11 & 76 & 3 & 37,2 & 73 & 6,3 & 43,2 & 7,48 \\
\hline 12 & 80,7 & 2,2 & 38,1 & 73,4 & 5,5 & 41 & 8,52 \\
\hline 13 & 82,5 & 2,8 & 39,8 & 76,8 & 4,7 & 42,3 & 6,51 \\
\hline 14 & 81,4 & 3,5 & 43,8 & 73,7 & 5,8 & 44 & 8,04 \\
\hline 15 & 76,9 & 3,3 & 38,7 & 74,7 & 5,6 & 40,7 & 3,76 \\
\hline 16 & 80 & 2,5 & 37,8 & 76 & 5,1 & 41,6 & 6,10 \\
\hline 17 & 82,5 & 2,8 & 38,4 & 73,5 & 5,8 & 40,9 & 9,81 \\
\hline 18 & 79,7 & 4 & 38,4 & 74 & 4,5 & 40,4 & 6,06 \\
\hline 19 & 80,3 & 3,8 & 43,4 & 76,5 & 5 & 44,5 & 4,13 \\
\hline 20 & 80,8 & 2,8 & 39 & 77,1 & 4,5 & 41,7 & 4,89 \\
\hline Media & 80,49 & 3,03 & 39,39 & 74,54 & 5,39 & 42,18 & 7,30 \\
\hline
\end{tabular}

Tabla III. Variación de Color en coordenadas $L^{*}, a^{*}, b^{*}$ de discos de resina del grupo B.

\begin{tabular}{lccccccc}
\hline \multicolumn{7}{c}{ Grupo B } & \multicolumn{7}{c}{ Color después de } \\
inmersión en té & $\Delta \mathrm{E}$ \\
& \multicolumn{5}{c}{ Color Basal } & \multicolumn{5}{c}{$\mathrm{L} 2$} & $\mathrm{a} 2$ & $\mathrm{~b} 2$ & \\
\hline 1 & $\mathrm{~L} 1$ & $\mathrm{a} 1$ & $\mathrm{~b} 1$ & & \\
2 & 80,2 & 3,7 & 35,5 & 76,1 & 5,2 & 39,2 & 5,72 \\
3 & 76,8 & 1,6 & 31,6 & 71,7 & 6,4 & 43,3 & 13,64 \\
4 & 74,4 & 3,5 & 33,8 & 74 & 3,5 & 39,4 & 5,61 \\
5 & 80,1 & 1,9 & 38,6 & 74,9 & 3,9 & 39,1 & 5,59 \\
6 & 78,7 & 1,6 & 38 & 75,7 & 4,5 & 40,7 & 4,47 \\
7 & 79,6 & 3,8 & 40,7 & 72,6 & 5,8 & 40,4 & 7,29 \\
8 & 77,7 & 2,1 & 33,2 & 79,9 & 4,5 & 40,7 & 8,18 \\
9 & 77,6 & 2,1 & 35,1 & 73,4 & 5,4 & 39 & 6,61 \\
10 & 79,9 & 2,5 & 33,3 & 74,8 & 5,2 & 41,6 & 10,11 \\
11 & 77,4 & 2,6 & 28,4 & 73,2 & 5,3 & 38,1 & 10,91 \\
12 & 80,1 & 3 & 34,4 & 79,1 & 4,3 & 39,7 & 5,55 \\
13 & 76,9 & 3,2 & 33,7 & 76,1 & 5,2 & 40,3 & 6,94 \\
14 & 74,9 & 2,3 & 31 & 72,1 & 5,5 & 38,9 & 8,97 \\
15 & 79,9 & 3,4 & 32,2 & 78,3 & 4,3 & 39,6 & 7,62 \\
16 & 79 & 3,2 & 35,2 & 77,8 & 4,8 & 41,4 & 6,51 \\
17 & 75,5 & 3 & 27,5 & 71,2 & 5,7 & 37,4 & 11,13 \\
18 & 79,3 & 2,2 & 36,5 & 75,7 & 4,3 & 40,1 & 5,51 \\
19 & 79,5 & 4,1 & 39,2 & 74,3 & 6 & 42,5 & 6,45 \\
20 & 75 & 2,7 & 32,6 & 72 & 5,8 & 41,7 & 10,07 \\
Promedio & 79,3 & 3,7 & 38,6 & 75,9 & 5,7 & 42,8 & 5,76 \\
& 78,09 & 2,81 & 34,46 & 74,48 & 5,07 & 40,30 & 7,63 \\
\hline
\end{tabular}


Tabla IV. Valores promedios de DE, mínimo/máximo, desviación estándar y P-Value.

\begin{tabular}{|c|c|c|c|c|c|}
\hline \multirow{2}{*}{$\begin{array}{l}\text { Grupos de resina } \\
\text { compuesta }\end{array}$} & \multicolumn{2}{|c|}{ Valores de $\Delta \mathrm{E}$} & \multirow{2}{*}{ Promedio $\Delta \mathrm{E}$} & \multirow{2}{*}{ Desviación } & \multirow{2}{*}{ T-Student P-Value } \\
\hline & Mínimo & Máximo & & & \\
\hline Grupo A & 3,76 & 13,26 & 7,30 & 2,76 & 0674 \\
\hline Grupo B & 4,97 & 13,64 & 7,63 & 2,39 & $0,0 / 4$ \\
\hline
\end{tabular}

Además, se les atribuye propiedades como mantener la estabilidad de color de la restauración mediante la reducción de acumulación de placa y la prevención de adhesión de pigmentos colorantes en restauraciones de composite, facilitando la limpieza de la restauración mediante la reducción de rugosidad superficial, e incrementando el brillo de la restauración (Aguilar et al., 2012). El objetivo de este estudio fue evaluar la capacidad de los selladores de superficie en base a resinas, de mantener el color de las resinas compuestas de restauración ante la acción de una bebida coloreada.

Los resultados obtenidos sugieren que la cobertura con sellador de superficie, a pesar de lograr la apariencia de una superficie suave y brillante, no incrementa la estabilidad de color de la resina restauradora ante la acción prolongada de soluciones colorantes. Estos resultados muestran que la inmersión por 7 días en una solución de té negro, genera un cambio de color (DE grupo A 7,30 y grupo $B$ 7,63 ) que es perjudicial en el color del material, provocando que esta variación supere los niveles de aceptabilidad. Habitualmente, se describe un DE de hasta 3,3 como límite para juzgar diferencias de color como clínicamente aceptables, siendo el ojo humano capaz de detectar cambios de DE igual a 1 (Chu et al., 2010; Takatsui et al., 2012).

De las variaciones de color observadas, también se puede inferir que bajo las condiciones experimentales, el té negro tuvo una fuerte capacidad de tinción, sin embargo, no se puede determinar si esto se debe a un efecto de adsorción, el cual consiste en un depósito superficial del colorante o a un efecto de absorción el cual se debería a una capacidad de penetración del colorante dentro de la matriz orgánica del composite. En términos generales, la sorción acuosa se correlaciona con el grado de conversión llevada a cabo por la polimerización y también con la naturaleza de la trama formada, tanto en términos de hidrofilicidad, estructura tridimensional y volumen libre (Pfeifer et al., 2011). Los componentes BisGMA y UDMA son moléculas de mayor hidrofilicidad que
TEGDMA y Bis-EMA (Gajewski et al., 2012) por lo que experimentarían una importante sorción acuosa, y es por ello que la solución de té negro, al estar en una solución acuosa podría tener un flujo importante hacia la estructura de la resina, y por lo mismo el sellador usado en este trabajo el cual consiste fundamentalmente de UDMA (Tabla I) no sería una barrera para el paso de la solución coloreada, es más sería un sustrato lábil para tincionarse. Sin embargo, en el presente estudio los discos revestidos de sellador no difirieron del grupo control en cuanto a tinción.

Con respecto a las implicancias clínicas de este trabajo, se debe considerar que una solución de té negro como la usada en esta investigación difícilmente estará en contacto prolongado con la resina selladora y restauradora, ya que normalmente en boca existen factores protectores de auto limpieza y dilución de soluciones como la saliva y la acción de los músculo de labio, lengua y mejilla, a lo que se debe sumar la función de barrido que realizan las técnicas de higiene del paciente. En el estudio de Bezgin et al. (2015) se determinó que el cepillado de especímenes inhibía la adsorción de colorantes en la superficie de los materiales restauradores y hacía decrecer la cantidad de cambio de color en ellos.

El resultado del presente trabajo confirma que la tinción de las resinas compuestas restauradoras depende no tan solo de las irregularidades de superficie, sino que también de la estructura del monómero (Gönülol \& Yilmaz, 2012), ya que en la situación experimental, tanto los discos perfectamente pulidos como los pulidos y protegidos con sellador de superficie, se tiñeron en gran magnitud, explicándose esto por la estructura y composición de éstos.

A la luz de los resultados, es posible que un sellador de superficie con distinta composición, ya sea con monómeros menos hidrofílicos o con algún porcentaje de relleno inorgánico pudieran ofrecer un mejor comportamiento como protector de cambios de color de restauraciones, dado que existen en el 
mercado diferentes formulaciones con distintos monómeros además de UDMA tales como: BisGMA, TEGDMA, THFMA, e incluso se han agregado algunos rellenos para mejorar propiedades mecánicas (Owens \& Johnson, 2006; Lopes et al., 2012). Al respecto, $\mathrm{S}$, ahin et al. evaluaron el efecto de distintos selladores de superficie ante la inmersión de discos de resina en una solución de café. Los resultados demostraron que existió un valor de DE menor en discos con selladores de superficie en relación al grupo control. Los selladores evaluados en este estudio fueron (Palaseal ${ }^{\circledR}$, Heraeus Kulzer $\mathrm{GmbH}$ Optiglaze $₫$, GC Corp Biscover $\AA$, Bisco Inc $®)$ (S, ahin et al.). Doray et al. también tuvieron resultados variables según el sellador de superficie usado, mostrando un bajo rendimiento el sellador en base a UDMA con relleno inorgánico Fortify Plus.

\section{CONCLUSIÓN}

A partir de los resultados obtenidos en este estudio experimental, es posible concluir que no existe diferencia significativa en el color de una resina compuesta con sellador de superficie vs. sin sellador de superficie después de someterlas a 7 días de inmersión en té negro. Por lo tanto, bajo los criterios y metodología empleada en este estudio, se acepta la hipótesis planteada.

AGUIRRE, P. A.; GALLEGOS, F. A.; BERSEZIO, M. C.; ESTAY, L. J. \& ARIAS, F. R. Resin-based surface sealants: potential to prevent exogenous staining. Int. J. Odontostomat., 12(4):348-354, 2018.

ABSTRACT: The objective of the present work was to study the potential of surface sealers based on resins to avoid color changes of resin composite restoration before the action of a colored drink. Therefore, a sample of 40 discs of nanocomposite Filtek Z350 XT (3M-ESPE) color A2D of $7 \times 2 \mathrm{~mm}$ was included, these were conventionally polished with a series of aluminum oxide discs and randomly distributed in 2 groups of 20 specimens. Group 1 was sealed with its entire surface using Fortify ${ }^{\circledR}$ sealant, group 2 was left untreated. All discs $(n=40)$ were subjected to a thermocycling process after which their initial color was measured by the Vita Easyshadem ${ }^{\circledR}$ point spectrophotometer. Each group of discs was immersed in metallic stainless steel containers, in a concentrated infusion of black tea for 7 days at $37^{\circ} \mathrm{C}$. Finally, the final color was measured in each of the disk groups following the immersion period. Results were subjected to the statistical analysis with the Stata 12 program and the T-Student test with a significance of $p<0.05$. The results indicated a significant color change in both groups of discs, being slightly lower in the group of sealed discs, however, there was no significant difference between them. In conclusion it was determined that under the conditions of the present study the use of the Fortify ${ }^{\circledR}$ surface sealant did not prevent the color change of the nanocomposite restoration resin.

KEY WORDS: composite resins, sealants, color.

\section{REFERENCIAS BIBLIOGRÁFICAS}

Aguilar, F. G.; Roberti Garcia, L. da F.; Cruvinel, D. R.; Sousa, A. B. \& de Carvalho Panzeri Pires-de-Souza, F. Color and opacity of composites protected with surface sealants and submitted to artificial accelerated aging. Eur. J. Dent., 6(1):24-33, 2012.

Avsar, A.; Yuzbasioglu, E. \& Sarac, D. The effect of finishing and polishing techniques on the surface roughness and the color of nanocomposite resin restorative materials. Adv. Clin. Exp. Med., 24(5):881-90, 2015.

Bagheri, R.; Burrow, M. F. \& Tyas, M. Influence of food-simulating solutions and surface finish on susceptibility to staining of aesthetic restorative materials. J. Dent., 33(5):389-98, 2005.

Bezgin, T.; Özer, L.; Tulga Öz, F. \& Özkan, P. Effect of toothbrushing on color changes of esthetic restorative materials. J. Esthet. Restor. Dent., 27 Suppl. 1:S65-73, 2015.

Catelan, A.; Briso, A. L.; Sundfeld, R. H.; Goiato, M. C. \& dos Santos, P. H. Color stability of sealed composite resin restorative materials after ultraviolet artificial aging and immersion in staining solutions. J. Prosthet. Dent., 105(4):23641, 2011.

Dietschi, D.; Campanile, G.; Holz, J. \& Meyer, J. M. Comparison of the color stability of ten new-generation composites: an in vitro study. Dent. Mater., 10(6):353-62, 1994.

Doray, P. G.; Eldiwany, M. S. \& Powers, J. M. Effect of resin surface sealers on improvement of stain resistance for a composite provisional material. J. Esthet. Restor. Dent., 15(4):244-9, 2003.

Douglas, R. D.; Steinhauer, T. J. \& Wee, A. G. Intraoral determination of the tolerance of dentists for perceptibility and acceptability of shade mismatch. J. Prosthet Dent., 97(4):200-8, 2009.

Ertas, E.; Güler, A. U.; Yücel, A. C.; Köprülü, H. \& Güler, E. Color stability of resin composites after immersion in different drinks. Dent. Mater. J., 25(2):371-6, 2006.

Gajewski, V. E.; Pfeifer, C. S.; Fróes-Salgado, N. R.; Boaro, L. C. \& Braga, R. R. Monomers used in resin composites: degree of conversion, mechanical properties and water sorption/solubility. Braz. Dent. J., 23(5):508-14, 2012.

Gönülol, N. \& Yilmaz, F. The effects of finishing and polishing techniques on surface roughness and color stability of nanocomposites. J. Dent., 40 Suppl. 2:e64-70, 2012.

Guler, A. U.; Yilmaz, F.; Kulunk, T.; Guler, E. \& Kurt, S. Effects of different drinks on stainability of resin composite provisional restorative materials. J. Prosthet. Dent., 94(2):118-24, 2005.

International Commission on Illumination. Guide on Interior Lighting. In: Publication CIE, no. 29.2. Paris, The Commission, 1986.

Kumari, R. V.; Nagaraj, H.; Siddaraju, K. \& Poluri, R. K. Evaluation of the effect of surface polishing, oral beverages and food colorants on color stability and surface roughness of nanocomposite resins. J. Int. Oral Health, 7(7):63-70, 2015.

Lee, Y. K. \& Powers, J. M. Combined effects of staining substances on resin composites before and after surface sealant application. J. Mater. Sci. Mater. Med., 18(5):685-91, 2007. 
Leibrock, A.; Rosentritt, M.; Lang, R.; Behr, M. \& Handel, G. Colour stability of visible light-curing hybrid composites. Eur. J. Prosthodont. Restor. Dent., 5(3):125-30, 1997.

Lopes, M. B.; Saquy, P. C.; Moura, S. K.; Wang, L.; Graciano, F. M.; Correr Sobrinho, L. \& Gonini Júnior, A. Effect of different surface penetrating sealants on the roughness of a nanofiller composite resin. Braz. Dent. J., 23(6):692-7, 2012.

Omata, Y.; Uno, S.; Nakaoki, Y.; Tanaka, T.; Sano, H.; Yoshida, S. \& Sidhu, S. K. Staining of hybrid composites with coffee, oolong tea, or red wine. Dent. Mater. J., 25(1):125-31, 2006.

Owens, B. M. \& Johnson, W. W. Effect of new generation surface sealants on the marginal permeability of Class $\mathrm{V}$ resin composite restorations. Oper. Dent., 31(4):481-8, 2006.

Pfeifer, C. S.; Shelton, Z. R.; Braga, R. R.; Windmoller, D.; Machado, J. C. \& Stansbury, J. W. Characterization of dimethacrylate polymeric networks: a study of the crosslinked structure formed by monomers used in dental composites. Eur. Polym. J., 47(2):162-70, 2011.

Ren, Y. F.; Feng, L.; Serban, D. \& Malmstrom, H. S. Effects of common beverage colorants on color stability of dental composite resins: the utility of a thermocycling stain challenge model in vitro. J. Dent., 40 Suppl. 1:e48-56, 2012.

Sahin, O.; Dede, D. Ö.; Körog lu, A. \& Yılmaz, B. Influence of surface sealant agents on the surface roughness and color stability of artificial teeth. J. Prosthet. Dent., 114(1):130-7, 2015.

Sarac, D.; Sarac, Y. S.; Kulunk, S.; Ural, C. \& Kulunk, T. The effect of polishing techniques on the surface roughness and color change of composite resins. J. Prosthet. Dent., 96(1):33-40, 2006.

Sarafianou, A.; losifidou, S.; Papadopoulos, T. \& Eliades, G. Color stability and degree of cure of direct composite restoratives after accelerated aging. Oper. Dent., 32(4):406-11, 2007.

Singh, S.; Shah, N.; Mandlik, J.; Nair, M.; Jaggi, S. \& Kanyal, K. The effect of finishing and polishing procedures on the surface roughness of composite resin materials: An in-vitro study. IIOAB J., 6(3):17-21, 2015.

Soares, L. E.; Cesar, I. C.; Santos, C. G.; De Cardoso, A. L.; Liporoni, P. C.; Munin, E. \& Martin, A. A. Influence of coffee on reflectance and chemistry of resin composite protected by surface sealant. Am. J. Dent., 20(5):299-304, 2007.

Subramanya, J. K. \& Muttagi, S. In vitro color change of three dental veneering resins in tea, coffee and tamarind extracts. J. Dent. (Tehran), 8(3):138-45, 2011.

Sun, T.; Shao, L. Q.; Yi, Y. F.; Deng, B.; Wen, N. \& Zhang, W. W. Infinite optical thickness of dentine porcelain of IPS E.max A color series. Nan Fang Yi Ke Da Xue Xue Bao, 31(2):259-61, 2011.
Dirección para correspondencia:

Roque Arias Fredes

Universidad de Chile

Santiago

CHILE

Email: roqueariasf@gmail.com

Recibido : 07-06-2018

Aceptado: 25-09-2018 